\title{
Interaktion Clopidogrel und PPI: geringe Praxisrelevanz
}

\author{
Die Aufregung, die eine methodisch \\ fragwürdige Studie zur Aufhebung \\ der Wirkung von Clopidogrel durch \\ Protonenpumpenblocker hervor- \\ gerufen hat, scheint nicht gerecht- \\ fertigt.
}

- Thienopyridine wie Clopidogrel oder Prasugrel gehören heute zur Standardtherapie von Patienten mit Zustand nach Myokardinfarkt, PTCA und Stentimplantation. Zur Prophylaxe gastrointestinaler Blutungen erhalten diese häufig auch Protonenpumpenblocker. Als Pro-Drugs werden sie über das Cytochrom-P450-Enzymsystem in ihre aktiven Metaboliten umgewandelt. Nachdem viele PPIs Cytochrom P450-2C19 hemmen, wurde gemutmaßt, dass die Wirkung von Clopidogrel durch die Gabe von PPIs aufgehoben werden könnte.

In der PRINCIPLE-TIMI-44-Studie wurden 201 Patienten, die sich einer elektiven perkutanen Koronarintervention unterzogen, randomisiert mit
Prasugrel oder Clopidogrel behandelt. In der TRITON-TIMI-38-Studie erfolgte eine ähnliche Therapie bei $13608 \mathrm{~Pa}$ tienten mit akutem Koronarsyndrom.

In der TIMI-44-Studie standen 26,4\%, in der TIMI-38-Studie 33,3\% der Patienten unter einer Therapie mit einem PPI (Omeprazol, Esomeprazol, Lansoprazol oder Rameprazol). Die Hemmung der Plättchenaggregation unter $20 \mu \mathrm{mol}$ ADP wurde $30 \mathrm{Minu}$ ten, zwei und sechs Stunden nach der Ladungsdosis von Clopidogrel bzw. Prasugrel, nach 18-24 Stunden, an Tag 15 und an Tag 29 gemessen. In der TIMI-44-Studie war die mittlere Hemmung der Plättchenaggregation bei den Patienten unter PPI sechs Stunden nach der Ladungsdosis von $600 \mathrm{mg}$ Clopidogrel signifikant niedriger als bei den nicht mit einem PPI behandelten Personen. Nach der Ladungsdosis von $60 \mathrm{mg}$ Prasugrel war ein derartiger Unterschied nicht zu beobachten. In der TIMI-38-Studie bestand im Lauf von sechs Monaten Follow-Up kein signifikanter Unterschied hinsichtlich kardiovaskulärer Mortalität, Apoplex, Myokardinfarkt oder Stentthrombose in den Gruppen mit und ohne PPITherapie.

\section{Kommentar}

Die Studie bestätigt zwar die theoretischen Überlegungen zur möglichen Aufhebung der Hemmung der Plättchenaggregation, zeigt aber gleichzeitig, dass dieser Effekt für die langfristige Therapie von Patienten mit Stentimplantation keine wesentliche Rolle spielt. Die Furcht vor einer möglichen Stentthrombose und einem Infarktrezidiv sollte die Ärzte nicht davon abhalten, bei gegebener Indikation zu einem PPI zu greifen.

H. S. FÜEßL =

- M. L. O'Donoghue et al.

Pharmacodynamic effect and clinical efficacy of clopidogrel and prasugrel with our without a proton-pump inhibitor: an analysis of two randomised trials. Lancet 374 (2009) 9694, 989-997

\section{... dann wölbte sich der Bauch vor}

— Bei einem 77-jährigen Mann bestanden seit zwei Tagen Schmerzen im Bereich des rechten Oberbauches und ein Exanthem. Die Untersuchung ergab den typischen Befund eines Zosters und der Mann wurde mit täglich 5 x 800 mg Aciclovir über sieben Tage hinweg behandelt.

Zwei Wochen später stellte sich der Patient wegen einer Vorwölbung im Bereich des Abdomens vor. Als wahrscheinlichste Diagnose ergab sich eine segmentale Zosterparese, eine ungewöhnliche, aber charakteristische Komplikation des Zosters. Dabei kommt es durch die Parese der

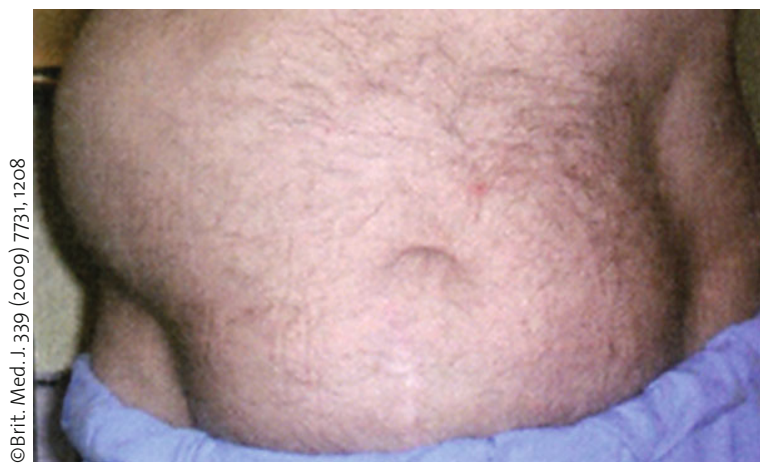

4 So kann sich der Bauch vorwölben, wenn es im Verlauf einer Zosterinfektion zu einer segmentalen Bauchmuskelparese kommt.

Abdominalmuskulatur zu einer Vorwölbung des Abdominalinhaltes, die bei den betroffenen Patienten große Beunruhigung auslöst. Die Patienten können insofern beruhigt werden, als sich dieser Zustand in der Regel im
Lauf mehrerer Monate wieder zurückbildet.

H. S. FÜEßL .

\footnotetext{
- M. A. Kordan

(Dudley Park Medical Centre, Birmingham B27 6OR, e-mail: mark.kordan@sbpct.nhs. uk). Brit. Med. J. 339 (2009) 7731, 1208
} 